\title{
Physical and Mechanical Properties of Transmission Line Galloping under the Action of Freezing and Thawing in Variable Temperature Range
}

\author{
Yu Ye $\mathbb{D}^{1,2} \mathrm{Li} \mathrm{Li}^{1,2}$ and Xunjian $\mathrm{Xu}{ }^{1,2}$ \\ ${ }^{1}$ State Grid Hunan Electric Power Company Disaster Prevention and Reduction Center, Changsha 410000, Hunan, China \\ ${ }^{2}$ State Key Laboratory of Disaster Prevention \& Reduction for Power Grid Transmission and Distribution Equipment, \\ Changsha 410000, Hunan, China
}

Correspondence should be addressed to Yu Ye; yuree2008@126.com

Received 6 July 2021; Revised 12 August 2021; Accepted 17 August 2021; Published 6 September 2021

Academic Editor: Sang-Bing Tsai

Copyright (c) 2021 Yu Ye et al. This is an open access article distributed under the Creative Commons Attribution License, which permits unrestricted use, distribution, and reproduction in any medium, provided the original work is properly cited.

\begin{abstract}
The western part of our country is mostly alpine regions. The rock and soil have been in a strong natural freeze-thaw environment for a long time, and their physical and mechanical properties are easily affected by external loads and external surroundings. Changes due to the influence of the environment will inevitably produce freeze-thaw cycles, damage and destruction, expansion and fracture, etc., resulting in more stable factors than usual. However, there is a lack of theoretical and practical experience in freeze-thaw rocks, especially freeze-thaw hard rocks. Therefore, studying the physical and mechanical properties and damage characteristics of rocks in alpine regions under freeze-thaw cycles has important significance. This paper uses dacite in the alpine region to carry out a freeze-thaw cycle experiment in a variable temperature range. Freezing and thawing cycle test, uniaxial compression test, triaxial compression test, and electron microscope scanning of the rock in the indoor saturated state were carried out. Combining theory with experimental mechanics, freeze-thaw mechanics, and damage mechanics, we studied freezethaw cycle in three variable temperature ranges $\left(-20^{\circ} \mathrm{C}-15^{\circ} \mathrm{C} ;-30^{\circ} \mathrm{C}-15^{\circ} \mathrm{C} ;-40^{\circ} \mathrm{C}-15^{\circ} \mathrm{C}\right)$, along with the physical and mechanical properties and damage characteristics of freeze-thaw dacite in the alpine region under cycling. The damage curve of the final theoretical model gradually approaches 1.0 with the increase of strain during the actual test. The rock sample after the medium failure still has a certain bearing capacity, and the rock sample is often destroyed before it reaches the theoretical failure strain.
\end{abstract}

\section{Introduction}

In general, frozen soil usually contains frozen soil and frozen rock. Frozen soil is a rock and soil with a temperature not exceeding $0^{\circ} \mathrm{C}$ and containing ice on the surface $[1-3]$. Most people believe that frozen soil and frozen rock coexist; in order to make the relevant research more detailed, it is necessary to distinguish the study of frozen soil and frozen rock [4]. Since China's reform and opening-up, especially in the past two decades, we have been vigorously carrying out strategic engineering construction and infrastructure construction. For example, the frost heave of rock reservoirs in petroleum reservoirs affects the safety of storage tanks and the frozen and expanded cracks of traffic tunnels [5]. The freezing and thawing of rock slopes and even landslides, the construction of railways and highways in high and cold regions, the construction of West-East Gas Pipeline, the construction of Sino-Russian oil and gas pipelines, the construction of many deep tunnels in high and cold regions, and the frost-thrusting of rock foundations due to freezing and thawing are a once-in-a-lifetime opportunity for scholars who study related content [6-9]. The alternating hot and cold changes in the alpine region have caused the freezing and thawing cycles of the rock mass in the freezing and thawing environment, and the damage has brought extremely serious impacts on a large number of engineering constructions [10]. At present, the stability evaluation of rock mass engineering in cold regions and the prevention and control of freeze-thaw disasters have become crucial scientific issues that need to be solved urgently [11-14]. 
Therefore, under the premise of China's resource development strategy moving westward $[15,16]$, the physicomechanical properties of rock under the temperature-temperature interval freeze-thaw cycle are studied, which has very important theoretical and practical value for resource development strategy $[17,18]$.

At present, there are two main types of monitoring systems for the settlement of poles [19], namely, the inclination monitoring system of the pole and tower based on the inclination sensor and the settlement monitoring system of the pole and the tower based on the BeiDou differential positioning technology [20]. The literature uses the inclination sensor on the top of the pole to monitor the inclination of the pole in real time, using the inclination of the pole and tower to judge the operating status of the pole and tower. The settlement monitoring of the pole and tower based on BeiDou differential positioning technology is obtained by the front-end monitoring device through the BeiDou terminal to obtain the position coordinates of the pole and tower, and the settlement value of the pole and tower is calculated through coordinate conversion. The cumulative effect of freeze-thaw damage keeps the elastic modulus of the concrete constant or slightly increasing, and the peak strain becomes smaller. The elastic modulus loss of concrete caused by ultralow temperature freeze-thaw cycles has good correspondence with the peak strain increase caused by cooling. Whether the upper limit temperature of the ultralow temperature freeze-thaw cycle is restored to normal temperature has little effect on the compressive deformation performance of concrete. The laser ranging sensor is arranged at a distance of $2 \mathrm{~m}$ from the tower leg to measure the distance to the laser ranging target. The laser ranging sensor communicates with the monitoring host through LoRa. When the laser ranging sensor measures the distance between the targets, the distance value is sent to the monitoring host through LoRa for data processing, and the settlement value of the tower leg is obtained. The monitoring host sends the tower settlement data and realizes real-time data display of the settlement value of the pole and tower leg, so as to realize the monitoring of the settlement of the pole and tower.

The application of numerical simulation technology in slope stability analysis is becoming more and more popular. Currently, numerical simulation methods are used to simulate the freeze-thaw stability of slopes. The mechanical properties directly imparted to the rock after freezing and thawing result in the calculated unstable slope being basically a freeze-thaw interface. However, this is not the case. It is especially suitable for rock slopes with large water content and weak structural development. This is because the freezing temperature of winter slopes in cold regions increases with depth. Therefore, it is unreasonable to assign the same parameters to the rock mass above the freeze-thaw interface during the calculation, and it is unreasonable to make calculation according to the same freeze-thaw conditions. According to the temperature gradient distribution characteristics of frozen rock layers in winter, different rock mass characteristics are assigned, which is a problem often overlooked in the stability analysis of slopes under the influence. It can be seen that there are still some problems in the application of experimental results of rock freezing and thawing cycles in cold mining areas. The study of rock physical and mechanical properties under variable temperature interval freeze-thaw cycles is a necessary condition for slope stability analysis under freezing and thawing conditions. However, little research has been done on the physical and mechanical properties of rocks under freezing and thawing in different temperature ranges.

In this paper, the Yingan rock in the high and cold area is taken as the research object. Through the simulation of indoor rock physics, mechanics, and freeze-thaw test, combined with the theory of experimental mechanics, freeze-thaw mechanics, and damage mechanics, the comparison, statistics, and analysis of various test results are performed. The research summarizes the changes of rock physical and mechanical properties under the action of freezing and thawing cycles in variable temperature interval, and based on the existing theoretical research on damage mechanics at home and abroad, the corresponding parameters or variables are selected for this test setup. However, the two existing methods have their own shortcomings. The inclination monitoring of the pole and tower based on the inclination sensor can only indirectly reflect the settlement state of the pole and tower, cannot quantify the settlement value of the pole and tower, and is limited by a single parameter. Based on the BeiDou differential positioning technology, the tower settlement monitoring error is too large, about $\pm 4.5 \mathrm{~cm}$, and the base station needs to be built during the implementation process, which is expensive and cannot be used in a large area at present. In view of the various problems of the existing transmission tower settlement monitoring, the article proposes a distance measurement based on the laser. The online monitoring system for the settlement of the power transmission line tower in the coal mine goaf can directly quantify the settlement value of the tower leg, the monitoring accuracy is high, and the error is within $\pm 2 \mathrm{~mm}$. Through the above research, the physicomechanical properties and damage characteristics of the frozen-thawed Yingan rock in the variable temperature interval are discussed. The effects of the freeze-thaw factors in the cold temperature range on the formation mechanism of the fragmented loose rock mass in the study area are briefly analyzed.

\section{Proposed Method}

\subsection{Freezing and Thawing}

2.1.1. The Concept of Freezing and Thawing. It mainly exists in glacial action areas, high mountains, and permafrost regions. The process freezing and thawing causes damage to the rock; loose deposits sway and are re-sorted to form a variety of frozen landforms. As the seasons alternate, freezing and thawing will occur repeatedly. During this process, the wedge opening pressure of the water film in the fine soil and mineral micro-cracks also changes. This results in the destruction of fine soils and minerals, which results in smaller particle sizes. It is different from the so-called cold 
weathering. The latter is caused by large cracks in the rock and wedge opening pressure of the ice in the void. In nonfrozen soils, the final weathering grain size of quartz is greater than that of rock. However, in the frozen soil area, due to cold hydration and weathering, the final weathering particle size of quartz $(0.05 \sim 0.01 \mathrm{~mm})$ is smaller than the final weathering particle size of feldspar $(0.1 \sim 0.05 \mathrm{~mm})$. Another physicochemical action that occurs during repeated freeze-thaw cycles is the agglomeration of colloids and cosmids into microaggregates, which increases the particle size of the rock. The effects of the above two different directions form similar results, so the content of rock powder particles undergoing repeated freezing and thawing is greatly increased. At the same time, a series of special cold landforms, namely, the ice edge, were formed. The ecosystems of alpine meadows and alpine swamp meadows are closely related to the frozen soil environment. The high ice content and low ground temperature of frozen soil are alpine meadows, alpine swamp meadows provide the basis for development, and the process of soil freezing and thawing is also obviously affected by changes in vegetation cover. When the upper limit of frozen soil depth increases, the coverage and biological production of alpine meadows and grasslands are significantly reduced. Freeze-thaw effects include freezethaw weathering, freeze-thaw disturbances, and freeze-thaw mud flow.

(1) Freeze-Thaw Weathering. Freeze-thaw weathering refers to the water in the cracks of the soil layer or rock formation. When the temperature drops, the rock rises and the rock near the crack is crushed into stones or smaller substances due to the pressure generated by the freezing expansion. It is a very common form of freeze-thaw action in alpine frozen soil areas.

(2) Freeze-Thaw Disturbance. Freezing and thawing disturbance refers to the plastic deformation phenomenon of the soil layer structure caused by freezing and extrusion in the active layer of permafrost.

(3) Freeze-Thaw Mud Flow. The freeze-thaw mud flow refers to the melting of the loose soil layer when the upper portion of the frozen soil layer is thawed. This water-rich soil diversity is malleable and creeps along the slope under neutral action.

\subsection{Principles of Mechanics}

2.2.1. Damage Mechanics. Damage mechanics is an important part of solid mechanics. It mainly studies the macroscopic mechanical effects caused by the generation and development of internal defects and the process and law of material destruction. The rock is mainly composed of various mineral particles, pores, and cement particles in the mountain, and there are defects such as dislocations, shear bands, microvoids, and microcracks inside. These mesoscale structures are the classic manifestations of damage. The rock is visible to the naked eye due to the load, and the instability and damage are caused by the random distribution of the internal microcracks and the macroscopic behavior caused by the microcrack initiation, expansion, and penetration. Therefore, the use of damage theory to study objects such as rock with initial defects is currently recognized as the most desirable and most effective method. Based on classification from the perspective of research methods, damage mechanics include macroscopic damage mechanics, mesodamage mechanics, and micro-damage mechanics. The macroscopic method is also called the phenomenological method. It focuses on the impact of damage on the macromechanical properties of the material and the damage evolution process of the structure, without investigating the physical background of the damage and the meso-structural changes inside the material. The mesoscopic method starts from the mesoscopic structure of the material, distinguishes different damage mechanisms, and focuses on the physical mechanism of the damage process. The micro method is used to study the physical process of material damage on the micro scale of atoms or molecules and to derive the macro response of damage based on quantum statistical mechanics. Among them, the mesoscopic method is based on the mesostructure of the material, distinguishing different damage mechanisms and focusing on the physical mechanism of the damage process. This method explores the nature and laws of material damage by studying the physical processes of meso-structural changes and adopts some mechanical average method. The results of the mesostructured unit are reflected in the macroscopic properties of the material. The meso-method mainly studies the damage evolution process of mesostructures, such as microcracks, micropores, and shear bands. On the one hand, it ignores the too complicated microscopic physical process and avoids the tedious calculation of microscopic statistical mechanics; on the other hand, it contains the mesoscopic geometry of different materials. It provides a physical background for building damage variables and damage evolution equations. The phenomenological damage theory developed in recent years is based on a mesoscopic theory of damage mechanics; because of its macroscopic and mesoscopic studies between methods, macroscopic modeling and constitutive relationship research can also provide important help. Several difficulties in its research are as follows:

First, how to properly define the damage of rock and how to quantitatively describe the damage variable. The selected damage variable not only needs to be easily measured in the test, but also can better reflect the damage evolution law of rock.

Second, the establishment of the damage evolution model. It should not only describe the commonality of the near equilibrium state (static), but also describe the particularity of the near-destructive state.

Third, the correspondence between meso-damage evolution and macroscopic mechanical properties.

2.2.2. Selection of Damage Variables. During the damage process of the material or structure, the internal microcracks or voids interact with each other, and there is no isolated crack that controls the development state of 
the damage. Moreover, it is not possible to geometrically describe all cracks, and it is not possible to determine the stress field near the crack tip. Therefore, the mechanics regards the region containing many scattered microcracks as a local uniform field and considers the overall effect of the crack in the field. An attempt is made to describe the damage state of a uniform field by defining a field variable that is irreversibly related. This field variable is the damage variable. A laser ranging target is installed at the bottom of the tower leg. The laser ranging target consists of 2 reflectors: one is a vertical reflector, and the installation direction is perpendicular to the ground; the other is an inclined reflector, and the installation direction is an angle of $60^{\circ}$ formed with the ground. The inclined reflector and the vertical reflector form a rightangled triangle in the same plane. After the tower has subsided, the freedom of the tower leg is affected by the binding force of the anchor bolt on the tower leg. In damage mechanics, the damage variable actually acts as a "degenerate operator," and these damage variables with objective statistical characteristics describe the damage state of the material or structure. From a thermodynamic point of view, the damage variable is actually a state variable inside the material, which reflects the irreversibility of the material structure change.

Damage can cause changes in the microstructure of the material and changes in some macroscopic physical properties, so the damage variable can be selected from both microscopic and macroscopic perspectives. The number, length, area, and volume of cracks can be selected at a microscopic angle; elastic modulus, yield stress, tensile strength, density, etc. can be selected from a macroscopic angle. Different damage variables can be selected for different damage processes, and even different damage processes can be selected for different damage variables. Depending on the difficulty of the research object and the way the mechanics are described, the damage variable can be defined as a scalar, vector, or tensor. For example, in the case of an isotropic distribution of microcracks, the damage variable can be in the form of a scalar form; for the case where the microcracks are regularly distributed in a plane, a vector perpendicular to the crack can be used to represent the damage; for the anisotropy of the microcrack, in the case of distribution, the damage variable can take the form of a tensor. Although the tensor indicates that the damage can more accurately reflect the arrangement state and mechanical properties of the microcrack, its mathematical expression is more complicated and more difficult in engineering applications. The ideal damage variable should have the following characteristics:

(1) The description of the damage is sufficiently accurate, and the description may be based on mesoscopic properties, such as the geometry, orientation, and configuration of the microcracks or microvoids.

(2) In order to facilitate the calculation of relevant mathematical operations and experimental results, experimental parameters of independent materials must be reduced as much as possible.

(3) The variable should have a certain physical or geometric meaning.

2.2.3. Effective Stress in the Rock Mass. In the context of the in-depth advancement of the power market reform, this paper uses a high-proportion hydropower transmission network as the carrier to update the market-oriented transaction signals to update the grid expansion planning research and proposes a high-proportion hydropower transmission grid expansion planning model that takes into account the electricity price signal orientation. The plan proposed in this paper is guided by market law signals and takes into account the price dynamics of transaction nodes. While promoting the consumption of hydropower resources, it can effectively alleviate network pressure and drive demand growth. System example analysis proves that the proposed expansion planning model has increased power grid investment income, social electricity costs, and realized social welfare benefits. At the same time, through the analysis of transaction signals such as network surplus, the model can reduce some actual positioning of the grid section, and at the same time, the different impacts on social power consumption will be combined. Once further indepth research is conducted on the mathematical properties of the actual transmission network planning problem model, more practical cases will be proposed for model application. The water in the pores of the rock body bears or transmits pressure, which can be called pore pressure. The load transmitted between the rock mass particles is called the effective stress. The Biot effective stress principle is generally used in the calculation. This principle states that the stress is negative when the positive pressure is pulled, the pore pressure is positive in the saturated region, and the pore pressure is negative in the unsaturated region.

\subsection{Mechanical Model of Rock Mechanics}

2.3.1. Establishment of Freeze-Thaw Stress Model. Since the research in this paper is only loaded with freeze-thaw action, the force of freeze-thaw load can be described by the principle of strain equivalence; equivalent plastic strain is a physical quantity used to determine the position of the yield surface of a material after strengthening. Its calculation formula is the same as the fourth strength theoretical calculation formula for calculating equivalent stress; just change stress into strain. What we get in the experiment is the elastoplastic stress-strain curve of the material under uniaxial tension. In practice, the stress state of the structure is often the spatial stress state. When yielding, it is also the spatial yield. We can find it by calculating the equivalent plastic strain. The following formula shows the equivalent 
stress corresponding to the strain state after plastic yielding and the spatial stress state of the structure at this time:

$$
D_{m}=D+D_{n}+D D_{m}
$$

In (1), $D_{m}$ is the total force of freeze-thaw load, and $D$ and $D_{n}$ are calculated separately to obtain the evolution equation of total freeze-thaw force:

$$
D_{m}=1-\frac{E_{n}}{E_{o}} \exp \left[-\frac{1}{m} \frac{E^{m}}{E_{o}}\right] .
$$

Monitor the role of the sun acquisition node in the whole system, establish a full coverage sensor network, complete the wireless network, and complete data acquisition and calculation, Collected by the tilt sensor, the calculated poles and tower positions are searched, then collected, packaged, and sent to the monitoring center via optical fiber or $4 \mathrm{G}$ communication. The monitoring platform has functions such as real-time data display, trend graph analysis, remote update and upgrade, and data statistical analysis. Therefore, the relationship between stress and strain demonstrating the force under the action of freezing is as shown in the following formula:

$$
\sigma=E_{o}\left(1-D_{m}\right) \varepsilon=E_{n}\left[-\frac{1}{m}\left(\frac{\varepsilon}{\varepsilon_{o}}\right)^{m}\right] \varepsilon .
$$

Based on the LoRa wireless transmission technology, the wireless transmission of the sensor is realized, and there is no need to consider the fixing of the signal line during installation.

\subsubsection{Force Evolution Equation under Rock Triaxial} Compression. The system consists of a laser ranging sensor, a monitoring host, and a monitoring center to form an overall monitoring camera. In the laser ranging sensor, first a laser diode is aimed at the target and emits laser pulses. The laser light is scattered in all directions after being reflected by the target. Part of the scattered light returns to the sensor receiver and is imaged on the avalanche photodiode after being received by the optical system. The monitoring distance sensor is the most direct element to collect information on the tower leg site. Its measurement accurately determines the accuracy of the entire monitoring system. The monitoring sensor being tracked will involve the contact work of the signal transmission line. Precautions should be taken for equipment failure after the signal transmission line is in contact with the environment. The intensity probability of the micro-element can be expressed by the following formula under the action of strain:

$$
Q(\varepsilon)=\frac{m}{\alpha}\left(\frac{\varepsilon}{\alpha}\right)^{m-1} e^{-(\varepsilon / \alpha)^{m}}
$$

In (4), $\varepsilon$ represents the strain of the micro-body, and $\alpha$ and $m$ represent the control variables of the Weibull distribution. Under the action of the load, the existing fine cracks inside the rock sample began to gradually expand outward. The direct function of this is to cause additional deformation or restraint deformation of the structure.
Cumulative effect is a stimulus-response phenomenon, which refers to a phenomenon in which the reaction time is shortened as the stimulation time or the area of stimulation increases when the stimulus acts on the senses. Therefore, the force variable $D$ in the cumulative effect of strain can be expressed by the following equation:

$$
D(\varepsilon)=\int_{0}^{\varepsilon} Q(\varepsilon) \mathrm{d} \varepsilon=1-e^{-(1 / m)(\varepsilon / \varepsilon 0)^{m}} .
$$

In (5), the parameter $\varepsilon$ represents the strain of the rock sample. Usually, we can also determine the control variable of the Weibull distribution through the stress-strain relationship of the material and then calculate the distance to be measured. According to the ranging principle of the laser ranging sensor, the STM8S207 with a 32-bit positioning interface and a three-level architecture is selected. The main control chip realizes the system call by controlling the power module and sends the processed laser ranging sensor data to the monitoring host. It can be known from formula (3) that the three-dimensional damage constitutive model of the rock sample can be expressed by the following formula:

$$
\sigma=(1-D)\left(C_{0}\right)^{-1}: \varepsilon
$$

In (6), $\sigma$ represents the stress tensor, $\varepsilon$ represents the strain tensor, and $\mathrm{C}_{0}$ represents the flexibility tensor of the rock sample. Using equal confining pressure triaxial compression, we select $\sigma_{2}=\sigma_{3}, \varepsilon_{2}=\varepsilon_{3}$, and then the rock sample under equal confining pressure conditions can be calculated according to (5) and (6), as shown in the following formula:

$$
\sigma_{1}=E \varepsilon_{1} e^{-(\varepsilon / \alpha)^{m}}+2 v \sigma_{3} .
$$

In (7), the elastic modulus of the rock sample is represented by $E$ and the Poisson's ratio is represented by $v$.

To determine the control parameter and $m$, you must rely on the stress-deformation rate relationship curve mentioned earlier and determine these two parameters according to the conditions. Under the condition that the determination mode is already known, if the parameters $\alpha$ and $m$ are only functions of confining pressure, the result can be obtained as the following equation:

$$
\left\{\begin{array}{l}
m=\frac{\varepsilon_{f}-(1-2 v) \sigma_{2} / E}{\varepsilon_{f} \operatorname{In}\left(E \varepsilon_{f} / \sigma_{f}-2 v \sigma_{2}\right)}, \\
\alpha=\left[\varepsilon_{f}-\left((1-2 v) \sigma_{2} / E\right)\right]\left[\frac{m \varepsilon_{f}}{\varepsilon_{f}-\left((1-2 v) \sigma_{2} / E\right)}\right]^{1 / m} .
\end{array}\right.
$$

2.3.3. Establishment of Rock Damage Model. The load subjected to freezing and thawing is represented by (1) in the total damage. The two-stage total damage under load is calculated, and the load subjected to freeze-thaw under triaxial compression is calculated as in the following formula: 


$$
D m=1-\frac{E_{n}}{E_{0}} \exp \left[\left(\frac{\varepsilon_{1}}{\alpha}\right)^{m}\right] .
$$

Equation (9) can indicate the damage caused to the rock; after the correction is successful, the timer is turned on, and the device automatically enters the low battery mode; $m$ represents the voltage transformation function. When the monitoring host receives the request data command from the monitoring center or the timer reaches $4 \mathrm{~h}$, the edge node will send a power controller to turn on the sensor. After turning on the sensor power, the laser ranging sensor will collect the distance to the laser ranging target. The monitoring host computer calculates the tower settlement value according to the above-mentioned tower settlement calculation method. The tower settlement value is packaged and sent to the monitoring center. After the process is over, the monitoring device enters the low power consumption mode and waits for the next request command or the next timing period.

$$
\sigma_{1}=E_{0}\left(1-D_{m}\right) \varepsilon_{1}=E_{n} \exp \left[\left(\frac{\varepsilon_{1}}{\alpha}\right)^{m}\right] \varepsilon_{1}
$$

Among them, $D_{m}$ is calculated by (9). It can be known from (9) that if the time calibration of both parties is successful, the timer will be turned on and the device will automatically enter the low power consumption mode. When the monitoring host receives the request data command from the monitoring center or the timer reaches $4 \mathrm{~h}$, the edge node will send a power controller to turn on the sensor. After turning on the sensor power, the laser ranging sensor will collect the distance to the laser ranging target. The monitoring host computer calculates the tower settlement value according to the above-mentioned tower settlement calculation method. The tower settlement value is packaged and sent to the monitoring center. After the process is over, the monitoring device enters the low power consumption mode and waits for the next request command or the next timing period.

\section{Experiments}

3.1. Temperature-Dependent Interval Freeze-Thaw Mechanics Experiment. Firstly, the saturated water absorption rate of the rock sample and the wave velocity of the longitudinal wave were detected, and eight rocks with different indexes were selected as samples. It is understood that the tower precipitation monitoring method was designed, the tower settlement monitoring device was designed, and the Xi'an monitoring device was installed on the $110 \mathrm{kV}$ transmission tower of the university of engineering to test the performance of the system. The tower legs are lifted by a hydraulic jack at an amplitude of $5 \mathrm{~mm} /$ time to simulate the topography of the tower. Due to the limitations of the experimental platform, the tower can only be lifted up to $70 \mathrm{~mm}$. An upward reflector and a curved reflector are installed at the bottom of the tower, and the laser ranging position is $2 \mathrm{~m}$ away from the telescope's ranging target. The real-time collection of the distance from the laser to the target can calculate the pixel value of the tower. The sample was saturated with water by a vacuum pumping method, with a vacuum pressure of $100 \mathrm{kPa}$ and pumping time of 4 hours, and then immersed in water for 12 hours. The experimental equipment includes vacuum water storage equipment, rock mechanics testing machine, low temperature box, and electric blast drying oven.

3.2. Freeze-Thaw Cycle Experiment. Online monitoring system was used for transmission line measurement in coal mine goaf based on laser ranging sensor. Compared with the transmission tower azimuth monitoring system based on inclination sensor, the laser ranging method can directly measure and analyze the field measurement of the tower without calculation. Since the height of the tower is known, there is no need to be limited by parameters. The specific test plan is set as follows:

(1) Set the rock freezing temperature to $-40^{\circ} \mathrm{C}$; that is, the freezing and thawing interval is $-40^{\circ} \mathrm{C}-15^{\circ} \mathrm{C}$.

(2) The experiment was set to 10 hours for a freeze-thaw cycle. Compared with the above-mentioned monitoring method, the online monitoring system of the power transmission line tower site in the coal mining area based on the laser distance sensor only needs to determine the environmental value of the tower through the geometric shape. The data processing method is simple and quick, and it will not be within $\pm 2 \mathrm{~mm}$.

(3) The online monitoring system for power transmission line analysis based on laser ranging sensor has high measurement accuracy and data realization ability. The tower position monitoring system based on BeiDou positioning needs to perform multiple conversion calculations on the tower coordinates to obtain the analysis information of the tower, with a value of about $\pm 4.5 \mathrm{~cm}$.

(4) Put the rock sample into the water for 12 hours.

\section{Discussion}

\subsection{Analysis of Freezing and Thawing Experiment in Variable Temperature Intervals}

\subsubsection{Saturated Maximum Water Storage and Conversion} Rate. In this paper, 20 freeze-thaw experiments were carried out successively, and then the quality of the dryness and water saturation of the Yingan rock samples was measured. Then, the saturated maximum water storage and conversion rate of rock samples in different temperature intervals after different moving times are calculated, as shown in Figures 1 and 2 (the average value of the initial density of the Yingan rock used in this paper is $2.631 \mathrm{~g} / \mathrm{cm}^{3}$ ). It can be seen from the figure that the saturated maximum water storage and conversion rate of rock samples with a lower temperature limit of $-20^{\circ} \mathrm{C}$ are generally lower, and the saturated water absorption rate begins to stabilize when it rises to $0.27 \%$. The mass loss rate also began to stabilize after the eighth freezethaw experiment. The biggest change was for the group with 


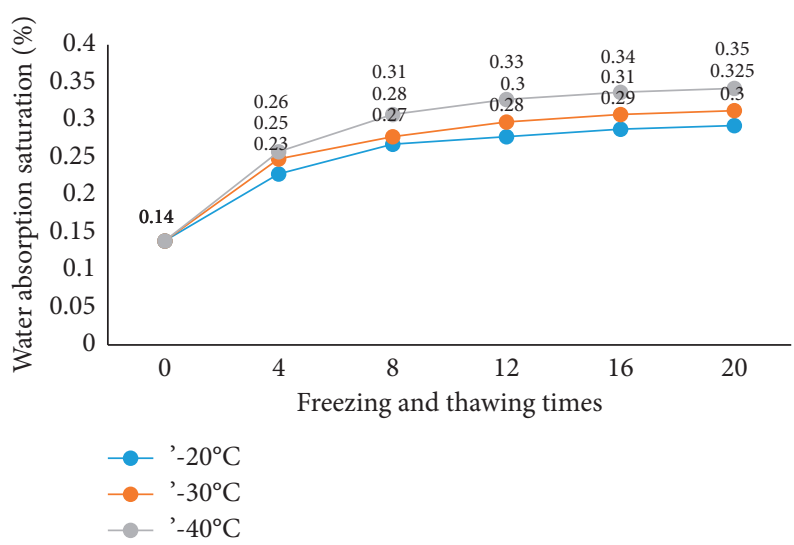

FIGURE 1: Rock sample water saturation rate.

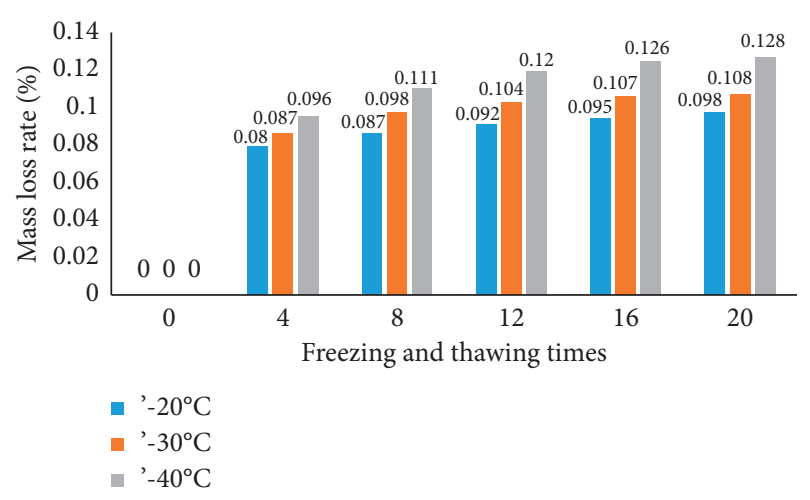

Figure 2: Mass loss rate of rock samples.

a temperature of $-40^{\circ} \mathrm{C}$, but the saturated maximum water storage and conversion rate were only $0.345 \%$ and $0.128 \%$. Therefore, on the whole, the saturated maximum water storage and conversion rate of Yingan rock are not too large.

4.1.2. Analysis of Mechanical Properties. The residual microstrain value of the specimens with different crack elongation is close. As the number of freeze-thaw cycles increases, the residual micro-strain value has an increasing trend, but the increase is much smaller than that of the cracked water-filled sample as shown in Table 1. The typical stress-strain curve measured after the test is shown in Figure 3. The installation and operation of the online monitoring system for the settlement of transmission lines in the goaf of coal mines based on laser ranging sensors are very convenient. The laser ranging target only needs to be arranged at the bottom of the tower leg, and the laser ranging sensor is arranged on the distance to the laser ranging target. The target is within $3 \mathrm{~m}$. The inclination monitoring system of the transmission tower based on the inclination sensor requires the sensor to be arranged at the top of the tower and $2 / 3$ of the height of the tower, which is difficult to install. The transmission settlement monitoring system based on the BeiDou differential positioning of the tower requires the establishment of base stations and installation monitoring on the top and bottom of the tower. The station is very large and expensive. By analyzing the image, the stress-strain curve obtained by the three sets of rock samples is not particularly large in this experiment. In the group of experiments with low temperature limit after 20 freeze-thaw cycles, the stress-strain curve compaction phase of the sample was relatively extended.

4.2. Analysis of Damage Caused by Freezing and Thawing of Yingan Rock. As shown in Figure 4 (n indicates the number of freeze-thaw cycles), when the freeze-thaw damage is infinitely close to 1.0 , the strain values of the Yingan rock samples are not much different, about 0.074. The measurement results of the tower unit based on the laser ranging method are basically consistent with the results measured by the electronic latitude and longitude monitor, which proves that the on-site monitoring system of the goaf pole and tower based on the laser distance measurement method can be applied to the monitoring of the tower unit in the goaf. In the current online monitoring system for power transmission towers, the power transmission tower goaf monitoring system based on laser ranging sensors has the advantages of being able to directly measure the tower site values, high measurement accuracy, and fast data processing speed. The damage of the Yingan rock sample is mainly caused by the damage caused by uniaxial compression.

Parameters of Yingan rock sample 0 and parameter $m$ are the same as those in Figure 5 and are different according to the number of freeze-thaw cycles. When the host power is turned on and initialized, the monitoring host will send a time correction command to the monitoring center. If the time calibration prompt reply indicates that the time calibration has failed, the monitoring host will continue to send request to the monitoring center. If the time calibration fails for 3 consecutive times, this proves that there is a communication failure between the monitoring host and the monitoring center. The monitoring center requires technicians to perform maintenance. 
TABLE 1: Experimental results before and after freezing and thawing.

\begin{tabular}{|c|c|c|c|c|}
\hline & Lower temperature limit $^{\circ} \mathrm{C}$ & $\begin{array}{c}\text { Uniaxial compressive } \\
\text { strength (average) }(\mathrm{MPa})\end{array}$ & $\begin{array}{c}\text { Modulus of } \\
\text { elasticity (average) }(\mathrm{GPa})\end{array}$ & Poisson ratio \\
\hline \multirow[t]{2}{*}{ Before experiment } & & 120.17 & 44.98 & 0.18 \\
\hline & $-20^{\circ} \mathrm{C}$ & 100.67 & 39.58 & 0.2 \\
\hline \multirow[t]{2}{*}{ Posttest } & $-30^{\circ} \mathrm{C}$ & 91.43 & 35.14 & 0.21 \\
\hline & $-40^{\circ} \mathrm{C}$ & 83.08 & 30.25 & 0.24 \\
\hline
\end{tabular}

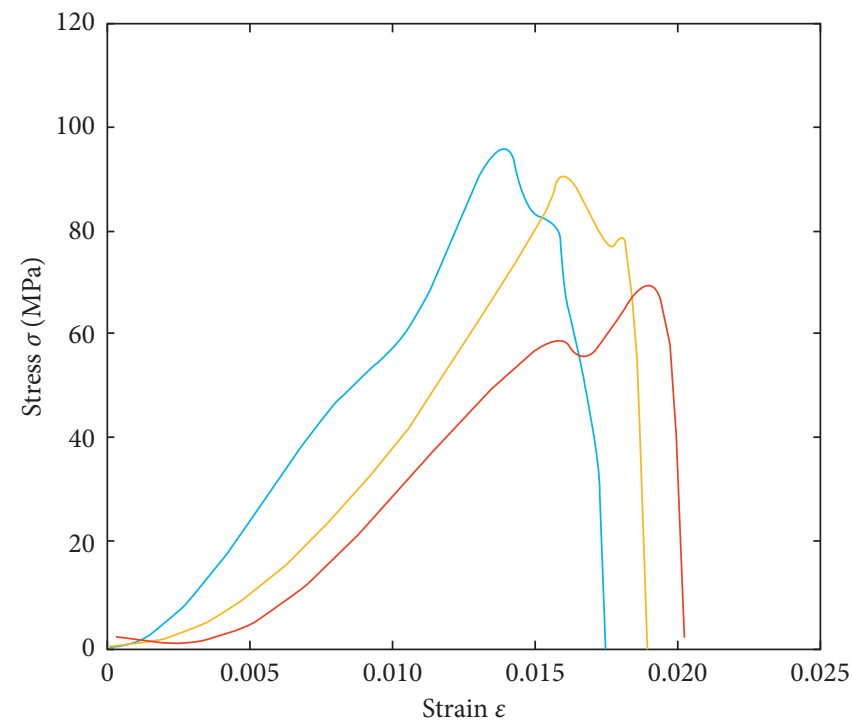

Lower temperature limit $-20^{\circ} \mathrm{C}$

Lower temperature limit $-30^{\circ} \mathrm{C}$

Lower temperature limit $-40^{\circ} \mathrm{C}$

FIgure 3: Typical stress-strain curve.

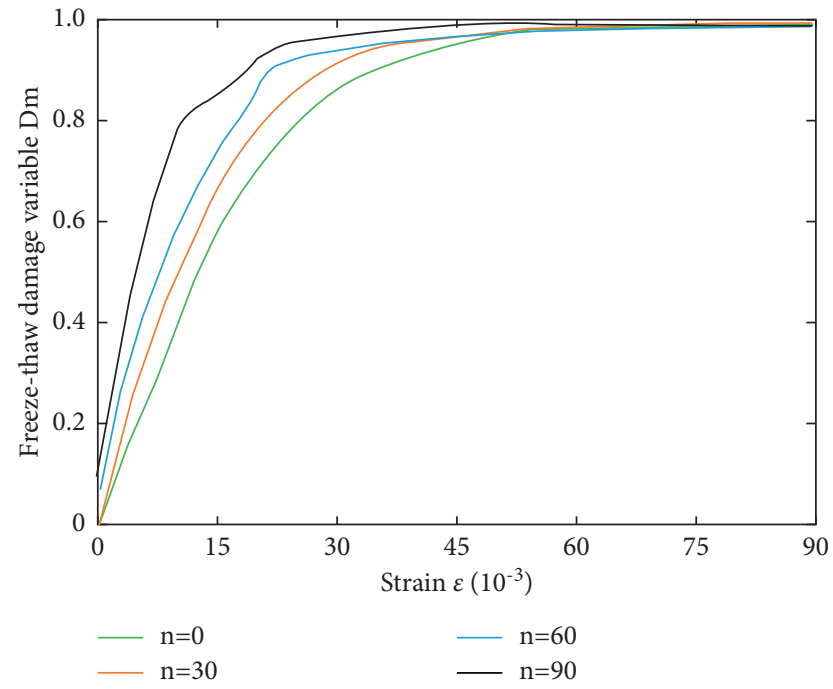

FIGURE 4: Damage curve of Yingan rock under freezing and thawing conditions. 


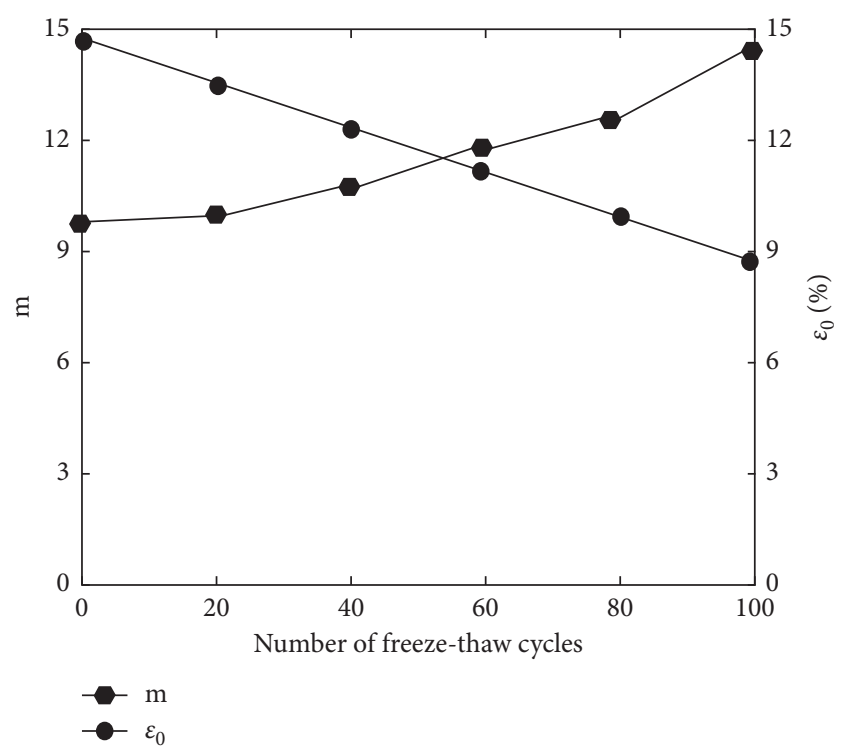

FIGURE 5: Curve of damage evolution parameters with freeze-thaw cycles.

\section{Conclusions}

This paper takes the Yingan rock in the high and cold area as the research object; simulates indoor petrophysical, mechanical, and freeze-thaw tests, combined with the theory of experimental mechanics, freeze-thaw mechanics, and damage mechanics; and then compares the test results. Through statistics and analysis, the research summarizes the changes of rock physical and mechanical properties under variable temperature interval freeze-thaw cycle, and based on the existing domestic and international theory of damage mechanics, the corresponding parameters are introduced for this test. We select appropriate mechanical correlation criteria and establish a constitutive model of the frozenthrust damage of the intact Yingan rock mass. The experimental results show that the mass loss rate, saturated water absorption rate, and peak strain of rock samples increase with the decrease of the lower limit of temperature. To sum up, it can be seen that freeze-thaw factors in high and cold areas have relatively limited freeze-thaw damage and deterioration of fresh dacite. The formation of a large number of fragmented loose rock masses in the study area is mainly due to the initial adjustment of the rock mass by the release of the unloading stress of the valley undercutting and is affected by the formation of rock. It is the result of comprehensive influences such as rock mass structure, plateau freezing and thawing weathering, and in situ stress. Therefore, the cold- and frost-resistant design of the dacite zone should also consider the influence of the following factors: the degree of early weathering and fragmentation of the local rock mass; the unloading and in situ stress.

\section{Data Availability}

This article does not cover data research. No data were used to support this study.

\section{Conflicts of Interest}

The authors declare that they have no conflicts of interest.

\section{Acknowledgments}

This project was supported by the State Grid Science and Technology of China (Grant no. 5216A01700UF) research on the prediction and application technology of winter ice wind temperature near the ground in the micro-topography of the power grid.

\section{References}

[1] C. P. Han, D. P. He, Y. M. Jia, and P. F. Cheng, "Attenuation law and reason analysis of resilience modulus of lime-improved subgrade soil under freeze-thaw cycle in variable temperature range," Highways, no. 9, pp. 14-18, 2015.

[2] M. Himdi and J. P. Daniel, "Analysis of printed linear slot antenna using lossy transmission line model," Electronics Letters, vol. 28, no. 6, pp. 598-601, 2018.

[3] W. Michal, K. Piotr, H. Da Mian, and H. Kacejko, "Overhead transmission line sag estimation using a simple optomechanical system with chirped fiber bragg gratings. part 1: preliminary measurements," Sensors, vol. 18, no. 1, pp. 309-310, 2018.

[4] C. Chen, B. Yang, S. Song, X. Peng, and R. Huang, "Automatic clearance anomaly detection for transmission line corridors utilizing UAV-borne LIDAR data," Remote Sensing, vol. 10, no. 4, pp. 613-616, 2018.

[5] S. Xiao, S. Liu, F. Jiang, M. Song, and S. Cheng, "Nonlinear dynamic response of reciprocating compressor system with rub-impact fault caused by subsidence," Journal of Vibration and Control, vol. 25, no. 11, pp. 1737-1751, 2019.

[6] R. Silva and S. Kurokawa, "Model of three-phase transmission line with the theory of modal decomposition implied," Energy and Power Engineering, vol. 5, no. 4, pp. 1139-1146, 2018.

[7] F. Fei, W. U. Gongping, M. Wang, Q. Cao, and S. Yang, "Robot delay-tolerant sensor network for overhead transmission line monitoring," Applied ences, vol. 8, no. 6, pp. 847-849, 2018.

[8] G. Pathirikkat, M. Balimidi, J. Maddikara, and M. Dusmanta Kumar, "Remote monitoring system for real time detection and classification of transmission line faults in a power grid using PMU measurements," Protection \& Control of Modern Power Systems, vol. 3, no. 1, pp. 16-19, 2018.

[9] Y. Deng, Z. He, F. Ling, S. Lin, L. Liu, and J. Zhang, "Research on fault location scheme for inverter AC transmission line of AC-DC hybrid system," IEEJ Transactions on Electrical and Electronic Engineering, vol. 13, no. 3, pp. 98-99, 2018.

[10] H. Song, D. Rawat, S. Jeschke, and C. Brecher, Cyber-Physical Systems: Foundations, Principles and Applications, Academic Press, Boston, MA, USA, 2016.

[11] H. Geng, Y. Huang, S. Yu, J. Yu, H. Hou, and Z. Mao, "Research on early warning method of overhead transmission line damage caused by typhoon disaster," Procedia Computer Science, vol. 130, no. 4, pp. 1170-1175, 2018.

[12] T. Hinge and S. Dambhare, "Synchronised/unsynchronised measurements based novel fault location algorithm for transmission line," IET Generation, Transmission \& Distribution, vol. 12, no. 7, pp. 1493-1500, 2018.

[13] N. Al-Areqi, K. Y. You, C. Y. Lee, N. H. Khamis, and M. N. Dimon, "Wideband and compact wilkinson power divider utilizing series delta-stub and folded stepped- 
impedance transmission line," Radioengineering, vol. 27, no. 1, pp. 200-206, 2018.

[14] Q. Guo, J. Xiao, and X. Hu, "New keypoint matching method using local convolutional features for power transmission line icing monitoring," Sensors, vol. 18, no. 3, pp. 66-69, 2018.

[15] Z. Lv and H. Song, "Mobile internet of things under data physical fusion technology," IEEE Internet of Things Journal, vol. 7, 2019 Nov 22.

[16] S.-B. Tsai, M.-F. Chien, Y. Xue et al., "Using the fuzzy dematel to determine environmental performance: a case of printed circuit board industry in taiwan," PLoS One, vol. 10, no. 6, Article ID e0129153, 2015.

[17] Z. Long, X. Huang, J. Jia, Y. Zhu, and W. Cao, "Detection of broken strands of transmission line conductors using fiber bragg grating sensors," Sensors, vol. 18, no. 7, pp. 2397-2401, 2018.

[18] M. Ba Hareh, F. G. Raul, and G. Ignacio, "E-textile embroidered metamaterial transmission line for signal propagation control," Materials, vol. 11, no. 6, pp. 955-959, 2018.

[19] M. Elhoseny, "Multi-object detection and tracking (MODT) machinelearning model for real-time video surveillance systems," Circuits, Systems, and Signal Processing, vol. 39, pp. 611-630, 2019.

[20] V. Chekh, D. Lyapunov, and A. Pravikova, "Mathematical model of power supply system for remotely operated underwater vehicle with dc power transmission line and load voltage feedback," IOP Conference Series: Materials Science and Engineering, vol. 19, no. 1, pp. 12-15, 2021. 